\title{
Lower Respiratory Tract Infections and Orofacial Clefts: A Prospective Cohort Study From the Japan Environment and Children's Study
}

Yukihiro Sato $^{1}$, Eiji Yoshioka ${ }^{1}$, Yasuaki Saijo ${ }^{1}$, Toshinobu Miyamoto ${ }^{2}$, Hiroshi Azuma ${ }^{3}$, Yusuke Tanahashi ${ }^{3}$, Yoshiya Ito $^{4}$, Sumitaka Kobayashi ${ }^{5}$, Machiko Minatoya ${ }^{5}$, Yu Ait Bamai ${ }^{5}$, Keiko Yamazaki ${ }^{5}$, Sachiko Itoh $^{5}$, Chihiro Miyashita ${ }^{5}$, Atsuko Ikeda-Araki ${ }^{5}$, Reiko Kishi ${ }^{5}$, and The Japan Environment and Children's Study (JECS) Group*

\footnotetext{
${ }^{1}$ Division of Public Health and Epidemiology, Department of Social Medicine, Asahikawa Medical University, Hokkaido, Japan ${ }^{2}$ Department of Obstetrics and Gynecology, Asahikawa Medical University, Hokkaido, Japan

${ }^{3}$ Department of Pediatrics, Asahikawa Medical University, Hokkaido, Japan

${ }^{4}$ Faculty of Nursing, Japanese Red Cross Hokkaido College of Nursing, Hokkaido, Japan

${ }^{5}$ Center for Environmental and Health Sciences, Hokkaido University, Hokkaido, Japan
}

Received September 8, 2020; accepted December 20, 2020; released online January 13, 2021

\begin{abstract}
Background: Lower respiratory tract infections (LRTIs) are a cause of inpatient and outpatient care among children. Although orofacial clefts seem to be associated with LRTIs, epidemiological studies are scarce on this topic. This study aimed to examine whether infants with orofacial clefts were associated with LRTIs.

Methods: This prospective cohort study used data from the Japan Environment and Children's Study, for which baseline recruitment was conducted during 2011-2014. This study included 81,535 participants. The number of infants with cleft lip and palate (CLP), cleft lip (CL), and cleft palate only (CP) was 67, 49, and 36, respectively. We defined history of LRTIs until 12 months' age reported by their mothers as the dependent variable. Accumulated breastfeeding duration was used as a potential mediator.

Results: The incidence proportion of LRTIs among the control group was $6.0 \%$. The incidence proportion among infants with CLP, CL, and CP were 11.9\%, 14.3\%, and 5.6\%, respectively. After adjusting for covariates, compared with the control group, infants with CLP and CL were associated with risk of LRTIs (incidence risk ratio [IRR] of CLP, 2.38; 95\% confidence interval [CI], 1.30-4.36 and IRR of CL, 2.73; 95\% CI, 1.40-5.33), but not ones with CP (IRR 1.08; 95\% CI, 0.28-4.15). Accumulated breastfeeding duration decreased the IRR of CLP only (IRR of CLP, 2.16; 95\% CI, 1.19-3.93).

Conclusions: Infants with orofacial clefts aged 1 year have a potentially high incidence proportion of LRTIs. Accumulated breastfeeding duration might mediate the associations of CLP.
\end{abstract}

Key words: cohort study; orofacial clefts; respiratory tract infection

Copyright $\odot 2021$ Yukihiro Sato et al. This is an open access article distributed under the terms of Creative Commons Attribution License, which permits unrestricted use, distribution, and reproduction in any medium, provided the original author and source are credited.

\section{INTRODUCTION}

Orofacial clefts are a common congenital anomaly, with approximately 1 case per 700 live birth. ${ }^{1,2}$ Particularly, this congenital anomaly has a higher incidence rate $(0.14 \%)$ in Japan. ${ }^{1,2}$ Orofacial clefts include cleft lip and cleft palate, and these conditions result from the lack of formations during embryogenesis development. ${ }^{3}$ As cleft lip and cleft palate can occur simultaneously or singly, orofacial clefts can be classified into three categories: cleft lip (CL), cleft palate only (CP), and cleft lip and palate (CLP).

Lower respiratory tract infections (LRTIs), mainly including pneumonia and bronchiolitis, have been a pervasive public health problem. ${ }^{4}$ There continues to be a considerable number of children as inpatients and outpatients due to LRTIs (the estimated number of inpatients and outpatients aged less than 1 year per day being 0.9 per 1,000 and 6.1 per 1,000 , respectively, in Japan). ${ }^{5}$ The occurrence of LRTIs in children can still lead to a large burden on healthy life, healthcare utilization, and costs. ${ }^{6,7}$ Previous studies have reported infection complications of the cleft palate due to the unfused secondary palate. The cleft palate causes nasopharyngeal closure dysfunction along with nasal regurgitation and a decrease in the oral suction. ${ }^{8}$ Inadequate airway protection during swallowing further triggers aspiration pneumonia. ${ }^{9,10}$ Therefore, mothers of infants with a cleft palate have reported feeding difficulties. ${ }^{10}$ Although breastfeeding is a

Address for correspondence. Yasuaki Saijo, Division of Public Health and Epidemiology, Department of Social Medicine, Asahikawa Medical University, 1-1-1 Midorigaoka higashi2-jo, Asahikawa, Hokkaido 078-8510, Japan (e-mail: y-saijo@asahikawa-med.ac.jp).

*The detail of JECS Group was shown in the acknowledge section. 
well-known preventive factor for respiratory infections, infants with a cleft palate have difficulty in breastfeeding from their mothers due to the unfused palate. ${ }^{11,12}$ The cleft palate may shorten the duration of breastfeeding and increase the risk of respiratory infections accordingly. Furthermore, infants with orofacial clefts tend to be born underweight. ${ }^{13,14}$ Due to impaired immune function from growth restriction, these infants can be at risk of hospitalization for pneumonia. ${ }^{15}$ Additionally, in Japan, cleft lip repair surgery is performed later than 3 months of age, and primary palatal surgery is performed between 12 and 24 months of age. ${ }^{16,17}$ Previous studies have reported that LRTIs can occur after cleft lip and palate surgery. ${ }^{18,19}$ Therefore, infants with cleft palate and cleft lip can have a high risk of LRTIs.

Although infants with oral clefts have been recognized as having risk factors for LRTIs, there seems to be a paucity of epidemiological evidence on the association between infants with orofacial clefts and LRTIs. To the best of our knowledge, only one study has reported these associations. A study of the Western Australian Register of Developmental Anomalies reported that infants with CLP and CP aged 0-2 years had an increased risk of hospitalization for any acute LRTIs (incidence rate ratio [IRR] of CLP, 2.5; 95\% confidence interval [CI], 1.2-4.8, and IRR of CP, 2.2 ; 95\% CI, 1.3-3.6). CL infants did not have this risk of hospitalization (IRR of CL, 0.5; 95\% CI, 0.1-1.6). ${ }^{20}$ However, that study used hospitalization as the outcome, not the incidence of LRTIs; therefore, it could only include severe cases. In addition, they did not examine potential pathways. Potential pathway analyses can help confirm and refute hypotheses. ${ }^{21}$ Thus, this study aimed to examine whether orofacial clefts in infants $\leq 12$ months of age were associated with the incidence of LRTIs using data from the Japan Environment and Children's Study (JECS). We also examined whether history of surgery under general anesthesia, accumulated breastfeeding duration, and birthweight mediated the associations.

\section{METHODS}

\section{Ethical approval}

Written informed consent was obtained from all participants and their proxies. The JECS protocol was approved by the Institutional Review Board of epidemiological studies of the Ministry of the Environment and Ethics Committees of all participating institutions. The JECS was conducted in accordance with the Helsinki Declaration and with other nationally valid regulations. $^{22}$

\section{Data sources and participants}

This prospective cohort study was conducted using a dataset, named jecs-an-20180131, from the JECS, the details of which are reported elsewhere. ${ }^{22,23}$ The JECS is a nationwide birth cohort study that aims to evaluate various environmental effects on children's health and development in Japan. The baseline recruitment was performed targeting early pregnant women in 15 areas between January 2011 and March 2014. Fifteen study areas were selected to cover the Japanese geographical areas from the north to the south. If individuals resided outside the study areas, then they could not participate in this survey.

Figure 1 shows a flowchart of the selection process of participants in this study. Because we excluded pregnant women who were participating for second time or more, 97,415 women participated in the baseline recruitment for the first time. After excluding 949 mothers who had multiple births, 96,466 mothers who had delivered a single birth remained. Among them, 2,244 had missing birth information and 1,432 had stillbirths or miscarriages; consequently, we observed a total of 92,790 single live births. We excluded 11 pairs because the sex of infants was not recorded, and 13 infants had non-classified orofacial cleft cases. In addition, to restrict nonsyndromic cases and nonaffected controls, we excluded 2,049 infants with other major congenital anomalies or syndromes. We also excluded 9,182 mothers who did not respond to the questionnaire when their infants were a year old. Thus, the final analyzed population included 81,535 pairs (mother-infant).

\section{Independent variables: cleft lip and palate, cleft lip, and cleft palate only}

We defined CLP, CL, and CP as independent variables. We obtained information on congenital anomalies from the medical record transcripts both at delivery and at 1 month of age. ${ }^{24} \mathrm{~A}$ previous study of JECS has described the details of the validation of the diagnosis process on congenital anomalies. ${ }^{24}$ We defined cases that were identified at either of the two periods after the definition of congenital anomalies in JECS. ${ }^{24}$ Orofacial cleft cases with an inconsistent diagnosis were defined as nonclassified orofacial clefts. ${ }^{24}$ Because we excluded infants with other major congenital anomalies or any syndromes, all included cases were defined as nonsyndromic. In addition, we also excluded nonclassified orofacial clefts. The control group included infants without any major congenital anomalies or syndromes.

\section{Dependent variable: lower respiratory tract infec- tions until 12 months of age}

We defined history of LRTIs until 12 months of age as the dependent variable. At 12 months of age, we obtained information on the history of LRTIs using a questionnaire answered exclusively by the mothers of these infants. The history of LRTIs was assessed based on the following question: "Has your child ever been diagnosed by a doctor for the following diseases, such as infectious diseases?" There was a checkbox in an infection disease section as "lower respiratory tract inflammations (such as bronchitis and pneumonia)." We defined checking the box of lower respiratory tract inflammation as having history of LRTIs until 12 months of age.

\section{Potential mediator variables}

According to previous studies, we considered history of surgery under general anesthesia, ${ }^{18,19}$ birthweight, ${ }^{13-15}$ and the accumulated breastfeeding duration ${ }^{10-12}$ as potential mediator variables. Due to lack of information on objective assessments of swallowing function and aspiration, we could not include them as variables. Information on surgery was obtained using the following question at 12 months of age: "How many of the following have happened since the birth of your child?" There was a section of "Surgery with general anesthesia." We obtained information on the number surgeries performed on the infant under general anesthesia until 12 months of age and defined the category as "none" and "at least once" accordingly. We also obtained information on birthweight from medical record transcripts at birth. Birthweight was classified as low birthweight $(<2,500 \mathrm{~g})$, normal birthweight $(2,500-4,000 \mathrm{~g})$, and high birthweight $(\geq 4,000 \mathrm{~g})$. Information on accumulated breastfeeding duration was also obtained from the checkbox question at 12 


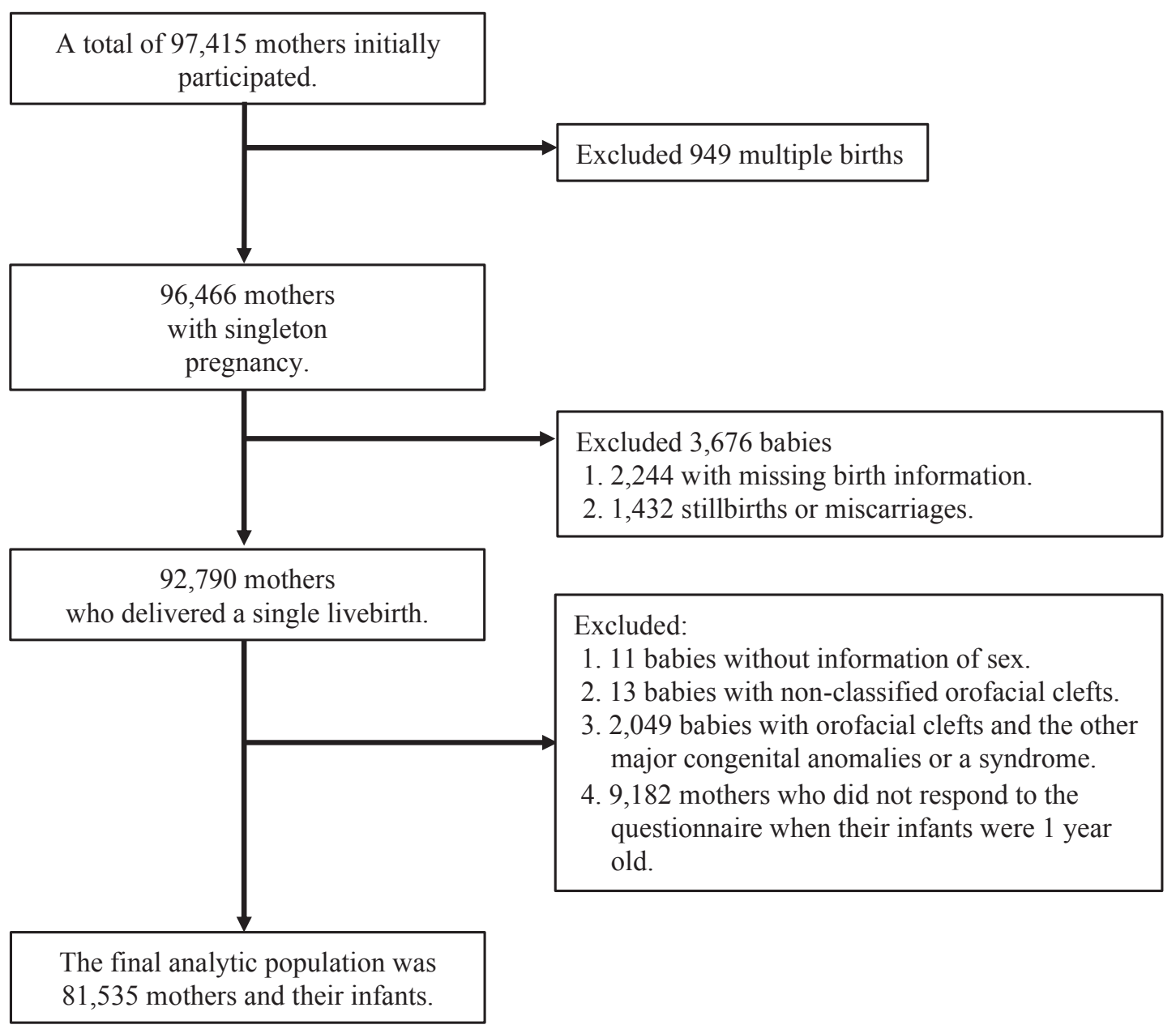

Figure 1. Flowchart of the selection of participants

months of age. Mothers checked each box of 1 month to 12 months of age according to when they had breastfed their infant. Then, we calculated the accumulated breastfeeding duration and divided it into three categories: 0 month, 1-6 months, and 7-12 months.

\section{Covariates}

The covariates were maternal age at delivery $(<25,25-29,30-34$, and $\geq 35$ years old); marital status during pregnancy (married and others); maternal active smoking status during pregnancy (never smoker, former smoker, and current smoker); frequencies of maternal passive smoking status during pregnancy (none, one to six times a week, and every day); maternal educational level (high school or lower, technical junior college or technical/vocational college, and university or higher); and annual household income ( $<4$ million yen, 4-6 million yen, 6-8 million yen, and >8 million yen); sex of the infants (male or female), season of birth (spring [March to May]; summer [June to August], autumn [September to November], and winter [December to February]); infant passive smoking status at 1 month of age (none, someone smoked in a separate room only, and someone smoked in a room with the infant); receipt of routine vaccines until 12 months of age in the National Immunization Program including Haemophilus influenzae type b, Pneumococcal, Hepatitis B virus, Diphtheria, Pertussis, Tetanus, Polio, and BCG (Bacille Calmette-Guérin) vaccines (received all vaccines and did not receive one or more); receipt of influenza virus vaccines (none or received); number of children living together at 1 year of age (none and $\geq 1$ ), and attendance in nursery school at 6 months of age (yes and no).

\section{Statistical analysis}

We conducted a Poisson regression analysis with a robust error variance to estimate IRRs for having a history of LRTIs with 95\% CIs. The IRRs can be interpreted as relative risks. ${ }^{25}$ The adjusted model included maternal age at delivery; marital status during pregnancy; maternal active smoking during pregnancy; frequencies of maternal passive smoking status during pregnancy; maternal educational level; annual household income during pregnancy; sex of the infant; season of birth; infant passive smoking status at 1 month of age; receipt of routine vaccines in the National Immunization Program at 1 year of age; receipt of influenza virus vaccines at 1 year of age; number of children living together at 1 year of age; and attendance in nursery school at 6 months of age. Then, we confirmed whether the directions of possible mediator variables matched to our hypothesis using stratified analyses (eTable 1). Therefore, we added only accumulated breastfeeding duration in the adjusted model to confirm the potential pathway. The percentage change by each potential mediator variable was calculated using the formula (IRR adjusted model $-I_{R R}$ adjusted model with a potential mediator variable $)$ / $\left(\mathrm{IRR}_{\text {adjusted model }}-1\right) \times 100 .{ }^{26}$ Two-sided $P$-values $<0.05$ were considered to indicate statistical significance. 
Because the data set had missing information, the k-nearest neighbor imputation method (the R package "DMwR") was used based on missing data at random for variables. ${ }^{27}$ eTable 2 shows the number of missing data for each variable. We also conducted an available-case analysis to confirm the validity of the results after imputation. All analyses were conducted using R (version 3.5.2; R Foundation for Statistical Computing, Vienna, Austria) on macOS.

\section{RESULTS}

This study included 81,535 participants. The median maternal age at delivery was 31.0 (the first and third quantiles were 28.0 and 35.0), and the proportion of female infants was $48.8 \%$. The characteristics of the participants according to the type of orofacial clefts are listed in Table 1. The number of infants with CLP, CL, and CP was 67, 49, and 36, respectively. Among the control group, the proportion of patients with history of surgery under general anesthesia was $1.1 \%$. The proportion of infants with CLP, CL, and CP was $87.9 \%, 85.4 \%$, and $6.1 \%$, respectively. The proportion of low birthweight cases in the control group was $7.8 \%$. The proportion of infants with low birthweight among those with CLP, CL, and CP was $17.9 \%, 22.4 \%$, and $8.3 \%$, respectively. Accumulated breastfeeding duration among $76.3 \%$ of infants in the control group was 7-12 months. Among $65.7 \%$ of infants with CLP, $69.4 \%$ of infants with CL, and $41.7 \%$ of infants with $\mathrm{CP}$, accumulated breastfeeding duration was 1-6 months, 7-12 months, and 1-6 months, respectively. In the control group, the proportion of infants with an incidence of LRTIs until 12 months of age was $6.0 \%$, and this incidence proportion among infants with CLP, CL, and CP was $11.9 \%$, $14.3 \%$, and $5.6 \%$, respectively.

Table 2 shows the associations between infants with orofacial clefts and LRTIs after imputation. In the crude model, compared with non-affected controls, infants with CLP and CL were significantly associated with LRTIs (IRR of infants with CLP, 2.00; 95\% CI, 1.04-3.83 and IRR of infants with CL, 2.39; 95\% CI, 1.20-4.75). Infants with CP was not significantly associated with LRTIs (IRR of infants with CP, 0.93; 95\% CI, 0.24-3.58). The adjusted model showed that, compared with the control group, infants with CLP and CL were associated with LRTIs (IRR of infants with CLP, 2.38; 95\% CI, 1.30-4.36 and IRR of infants with CL, 2.73; 95\% CI, 1.40-5.33), but not infants with CP (IRR of infants with CP, 1.08; 95\% CI, 0.28-4.15). The adjusted model with accumulated breastfeeding duration showed only a decreased IRR of infants with CLP (IRR of infants with CLP, 2.16; 95\% CI, 1.19-3.93 and percentage change $=15.9 \%$; IRR of infants with CL, 2.74; 95\% CI, 1.41-5.34 and percentage change $=-0.6 \%$; IRR of infants with $\mathrm{CP}, 1.04 ; 95 \% \mathrm{CI}$, $0.27-3.98$ and percentage change $=50.0 \%$ ). The results from an available-case analysis were consistent with those after imputation (eTable 3).

\section{DISCUSSION}

Among the control group, the incidence of LRTIs was $6.0 \%$. Among infants with CLP, CL, and CP, the incidence of LRTIs was $11.9 \%, 14.3 \%$, and $5.6 \%$, respectively. A history of surgery and birth weight were excluded due to the inconsistent directions of the associations. Accumulated breastfeeding duration decreased the IRR of CLP only. This study shows that infants with
CLP and CL had an increased risk of LRTIs after adjusting for covariates, while infants with $\mathrm{CP}$ did not.

This study showed that only infants with CP were not associated with increased risk. However, a previous study reported that infants with CLP and CP were associated with the risk of hospitalization for any acute LRTIs, but not CL. ${ }^{20}$ There could be potential reasons for this contradictory result. Moreover, both the current study and the previous study did not include the severity of orofacial clefts. In Table 1, the proportion of infants with low birthweight was relatively higher among CLP and $\mathrm{CL}$ cases than among $\mathrm{CP}$ cases. Previous studies reported low birthweight among CLP and CP cases but not among CL cases. $^{13,14}$ This result might reflect that the current $\mathrm{CP}$ cases included milder cases, such as Veau I, which might avoid aspiration and subsequent LRTIs. In addition, our study included only infants aged 12 months. In Japan, cleft palate surgery is generally performed after 12 months of age. As our study participants were 12 months of age, they should not be exposed to the risk of complications after surgery. Indeed, 93.9\% of infants with CP were not experienced any surgery under general anesthesia. There is still a possibility that $\mathrm{CP}$ infants would have an increased risk after 12 months of age. In addition, the CL cases might include more severe cases such as a complete cleft lip. Previous studies showed no significant association between CL and low birthweight. ${ }^{13,14}$ However, in this study, there was a larger proportion of infants with low birthweight among the CL cases than among the non-affected controls (22.4\% vs $7.8 \%)$. Further studies including a detailed classification of clefts are hence, needed to verify the validity of these results.

Although we conducted a mediation analysis, there was a limitation in the sample size. The models showed significant associations, yet they also presented with wide CIs, implying that the current results had a high degree of uncertainty. For example, the percentage change in infants with $\mathrm{CP}$ by accumulated breastfeeding duration was $50 \%$, and the IRR changed from 1.08 to 1.04 with wide CIs. When interpreting the mediation analysis, we should note a high degree of uncertainty in the results from the mediation analyses.

We considered four pathways: infectious complications of cleft surgery, low birthweight, shorter duration of breastfeeding, and aspiration due to the cleft palate. First, we could not conduct the mediation analysis using history of surgery due to the inconsistent directions of the associations. eTable 1 shows the low incidence of LRTIs among infants with orofacial clefts who had a history of surgery. This result means that history of surgery among infants with CLP or CL seems to be associated with the decreased risk of LRTIs. This is inconsistent with the results from recent studies, which reported a 1.9-8.9\% increase in respiratory complications among orofacial cleft patients after repair surgery. ${ }^{18,19}$ In this study, the time sequence between the outcome and surgery was lacked. Moreover, we could not collect details on surgery for clefts. Therefore, it is difficult to determine whether a repair surgery for clefts mediated the associations between infants with oral clefts and LTRIs. Further studies should the detailed information on surgery and severity for cleft lip. Second, birthweight also showed the inconsistent directions of the associations, although $17.9 \%$ of infants with CLP and $22.4 \%$ of infants with CL were born with low birthweight. eTable 1 shows that nonaffected infants with low birthweight had a low risk of LRTIs, whereas infants with orofacial clefts who were low birthweight had a high risk of LRTIs. Birthweight might not 
Table 1. Characteristics of the participants according to the type of orofacial clefts

\begin{tabular}{|c|c|c|c|c|c|c|c|c|c|}
\hline & & $\begin{array}{l}\text { Control } \\
(n=81,3\end{array}$ & $\begin{array}{l}\text { group } \\
383)\end{array}$ & $\begin{array}{l}\text { Infar } \\
\text { with } \\
(n=\end{array}$ & $\begin{array}{l}\text { CLP } \\
67)\end{array}$ & $\begin{array}{l}\text { Infa } \\
\text { with } \\
(n=\end{array}$ & $\begin{array}{l}\text { he } \\
=49)\end{array}$ & & $\begin{array}{l}\text { ants } \\
\text { h CP } \\
=36)\end{array}$ \\
\hline & & $n$ & $\%$ & $n$ & $\%$ & $n$ & $\%$ & $n$ & $\%$ \\
\hline Independent variables & & & & & & & & & \\
\hline Maternal age at delivery, years & $<25$ & 7,256 & 8.9 & 6 & 9.0 & 4 & 8.2 & 1 & 2.8 \\
\hline & $25-29$ & 22,264 & 27.4 & 24 & 35.8 & 9 & 18.4 & 15 & 41.7 \\
\hline & $30-34$ & 29,251 & 35.9 & 23 & 34.3 & 21 & 42.9 & 11 & 30.6 \\
\hline & $\geq 35$ & 22,608 & 27.8 & 14 & 20.9 & 15 & 30.6 & 9 & 25.0 \\
\hline Marital status during pregnancy & Married & 77,156 & 95.8 & 64 & 95.5 & 46 & 95.8 & 34 & 94.4 \\
\hline & Others & 3,407 & 4.2 & 3 & 4.5 & 2 & 4.2 & 2 & 5.6 \\
\hline Maternal active smoking during pregnancy & Never smoker & 47,941 & 59.7 & 38 & 56.7 & 25 & 52.1 & 23 & 63.9 \\
\hline & Former smoker & 29,111 & 36.2 & 28 & 41.8 & 19 & 39.6 & 12 & 33.3 \\
\hline & Current smoker & 3,284 & 4.1 & 1 & 1.5 & 4 & 8.3 & 1 & 2.8 \\
\hline Frequencies of maternal passive smoking status during pregnancy & None & 40,681 & 50.5 & 29 & 43.3 & 21 & 43.8 & 20 & 55.6 \\
\hline & One to six times a week & 25,762 & 32.0 & 26 & 38.8 & 15 & 31.2 & 12 & 33.3 \\
\hline & Every day & 14,093 & 17.5 & 12 & 17.9 & 12 & 25.0 & 4 & 11.1 \\
\hline Maternal educational attainment & High school or lower & 27,928 & 34.7 & 32 & 48.5 & 15 & 31.2 & 12 & 33.3 \\
\hline & $\begin{array}{l}\text { Technical junior college or } \\
\text { technical/vocational college }\end{array}$ & 34,324 & 42.7 & 16 & 24.2 & 25 & 52.1 & 18 & 50.0 \\
\hline & University or higher & 18,158 & 22.6 & 18 & 27.3 & 8 & 16.7 & 6 & 16.7 \\
\hline Annual household income during pregnancy & $<4$ million yen & 29,579 & 39.3 & 21 & 32.8 & 19 & 45.2 & 12 & 34.3 \\
\hline & 4-6 million yen & 25,165 & 33.4 & 22 & 34.4 & 12 & 28.6 & 11 & 31.4 \\
\hline & 6-8 million yen & 12,203 & 16.2 & 14 & 21.9 & 6 & 14.3 & 8 & 22.9 \\
\hline & $>8$ million yen & 8,341 & 11.1 & 7 & 10.9 & 5 & 11.9 & 4 & 11.4 \\
\hline Sex of the infant & Male & 41,669 & 51.2 & 46 & 68.7 & 29 & 59.2 & 15 & 41.7 \\
\hline & Female & 39,714 & 48.8 & 21 & 31.3 & 20 & 40.8 & 21 & 58.3 \\
\hline Season of birth & Spring (March to May) & 18,967 & 23.3 & 20 & 29.9 & 8 & 16.3 & 6 & 16.7 \\
\hline & Summer (June to August) & 20,820 & 25.6 & 12 & 17.9 & 9 & 18.4 & 11 & 30.6 \\
\hline & Autumn (September to November) & 22,490 & 27.6 & 20 & 29.9 & 22 & 44.9 & 11 & 30.6 \\
\hline & Winter (December to February) & 19,106 & 23.5 & 15 & 22.4 & 10 & 20.4 & 8 & 22.2 \\
\hline Infant passive smoking status at one month of age & None & 39,216 & 48.6 & 32 & 49.2 & 18 & 37.5 & 23 & 63.9 \\
\hline & Someone smoked in a separate room only & 39,655 & 49.1 & 32 & 49.2 & 29 & 60.4 & 13 & 36.1 \\
\hline & Someone smoked in a room with the infant & 1,863 & 2.3 & 1 & 1.5 & 1 & 2.1 & 0 & 0.0 \\
\hline Receiving routine vaccines in the National Immunization Program & Did not receive one or more & 27,971 & 34.4 & 27 & 40.3 & 24 & 49.0 & 13 & 36.1 \\
\hline at 1 year of age & Received all vaccines & 53,412 & 65.6 & 40 & 59.7 & 25 & 51.0 & 23 & 63.9 \\
\hline Receiving influenza virus vaccines at 1 year of age & None & 66,694 & 82.0 & 59 & 88.1 & 43 & 87.8 & 28 & 77.8 \\
\hline & Received & 14,689 & 18.0 & 8 & 11.9 & 6 & 12.2 & 8 & 22.2 \\
\hline Number of children living together at 1 year of age & None & 39,750 & 48.8 & 40 & 59.7 & 20 & 40.8 & 20 & 55.6 \\
\hline & $\geq 1$ & 41,633 & 51.2 & 27 & 40.3 & 29 & 59.2 & 16 & 44.4 \\
\hline Attending nursery school at 6 months of age & Yes & 21,464 & 26.5 & 11 & 16.4 & 6 & 12.2 & 9 & 25.0 \\
\hline & No & 59,571 & 73.5 & 56 & 83.6 & 43 & 87.8 & 27 & 75.0 \\
\hline Potential mediators & & & & & & & & & \\
\hline History of any surgery under general anesthesia & None & 67,686 & 98.9 & 8 & 12.1 & 7 & 14.6 & 31 & 93.9 \\
\hline & At least once & 759 & 1.1 & 58 & 87.9 & 41 & 85.4 & 2 & 6.1 \\
\hline Birthweight & Low birthweight $(<2,500 \mathrm{~g})$ & 6,307 & 7.8 & 12 & 17.9 & 11 & 22.4 & 3 & 8.3 \\
\hline & Normal birthweight $(2,500-4,000 \mathrm{~g})$ & 74,332 & 91.4 & 55 & 82.1 & 37 & 75.5 & 33 & 91.7 \\
\hline & High birthweight $(\geq 4,000 \mathrm{~g})$ & 696 & 0.9 & 0 & 0.0 & 1 & 2.0 & 0 & 0.0 \\
\hline Accumulated breastfeeding duration & 0 month & 2,124 & 2.6 & 10 & 14.9 & 3 & 6.1 & 9 & 25.0 \\
\hline & 1 to 6 months & 17,171 & 21.1 & 44 & 65.7 & 12 & 24.5 & 15 & 41.7 \\
\hline & 7 to 12 months & 62,088 & 76.3 & 13 & 19.4 & 34 & 69.4 & 12 & 33.3 \\
\hline Dependent variable & & & & & & & & & \\
\hline Lower respiratory tract infections until 12 months of age & & 4,863 & 6.0 & 8 & 11.9 & 7 & 14.3 & 2 & 5.6 \\
\hline
\end{tabular}

CL, cleft lip; CLP, cleft lip and palate; CP, cleft palate only.

${ }^{a}$ Routine vaccines in the National Immunization Program included Haemophilus influenzae type b, Pneumococcal, Hepatitis B virus, Diphtheria, Pertussis, Tetanus, Polio, and BCG vaccines.

mediate the associations. Third, the IRRs of infants with CLP decreased after adjusting for accumulated breastfeeding duration, whereas those of infants with CL did not (percentage change in infants with $\mathrm{CPL}=15.9 \%$ and that in infants with $\mathrm{CL}=-0.6 \%$ ). Breastfeeding can promote the infant's immune function and development of the respiratory tract. ${ }^{12}$ Previous studies have suggested that mothers of infants with a cleft palate tend to face feeding difficulties due to nasopharyngeal closure dysfunction and poor oral suction. ${ }^{10}$ In this study, a shorter duration of breastfeeding was observed among CLP and CP. The duration of breastfeeding may partially explain the association between infants with CLP only and LRTIs but not between infants with $\mathrm{CL}$ and LRTIs. Finally, we supposed that aspiration is an important mechanism of LRTIs for cleft palate. However, our assumption seems to be inconsistent because the current results show that infants with CLP had an increased risk, while infants 
Table 2. Associations between orofacial cleft status and lower respiratory tract infections after imputation

\begin{tabular}{|c|c|c|c|c|c|c|c|c|c|c|}
\hline & \multirow{3}{*}{$n$} & \multirow{3}{*}{ Incidence } & \multirow{3}{*}{ Incidence proportion (\%) } & \multirow{2}{*}{\multicolumn{2}{|c|}{$\begin{array}{l}\text { Crude model } \\
(n=81,535)\end{array}$}} & \multirow{2}{*}{\multicolumn{2}{|c|}{ Adjusted Model ${ }^{\mathrm{a}}$}} & \multicolumn{3}{|c|}{ Adjusted } \\
\hline & & & & & & & & $\begin{array}{l}\text { Mode } \\
\text { breas } \\
(n=\end{array}$ & $\begin{array}{l}\text { + Accumulated } \\
\text { eding duration } \\
535 \text { ) }\end{array}$ & $\begin{array}{l}\text { Percentage change } \\
\text { by accumulated } \\
\text { breastfeeding duration }\end{array}$ \\
\hline & & & & IRR & $95 \% \mathrm{CI}$ & IRR & $95 \% \mathrm{CI}$ & IRR & $95 \% \mathrm{CI}$ & \\
\hline Control group (reference) & 81,383 & 4,863 & 6.0 & 1.00 & - & 1.00 & - & 1.00 & - & \\
\hline Infants with CLP & 67 & 8 & 11.9 & 2.00 & $1.04,3.83$ & 2.38 & $1.30,4.36$ & 2.16 & $1.19,3.93$ & 15.9 \\
\hline Infants with $\mathrm{CL}$ & 49 & 7 & 14.3 & 2.39 & $1.20,4.75$ & 2.73 & $1.40,5.33$ & 2.74 & $1.41,5.34$ & -0.6 \\
\hline Infants with $\mathrm{CP}$ & 36 & 2 & 5.6 & 0.93 & $0.24,3.58$ & 1.08 & $0.28,4.15$ & 1.04 & $0.27,3.98$ & 50.0 \\
\hline
\end{tabular}

CI, confidence interval; CL, cleft lip; CLP, cleft lip and palate; CP, cleft palate only; IRR, incidence risk ratio.

${ }^{a}$ Adjusted Model included factors such as maternal age at delivery, marital status during pregnancy, maternal active smoking during pregnancy, frequencies of maternal passive smoking status during pregnancy, maternal educational attainment, annual household income during pregnancy, sex of the infant, season of birth, infant passive smoking status at one month of age, receiving routine vaccines in the National Immunization Program at 1 year of age, receiving influenza virus vaccines at 1 year of age, number of children living together at 1 year of age, and attending nursery school at 6 months of age.

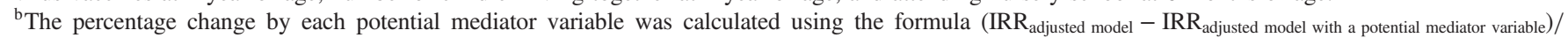
$\left(\mathrm{IRR}_{\text {adjusted model }}-1\right) * 100$.

The imputation was conducted using the k-nearest neighbor imputation method.

with CP did not. In addition, infants with CL had a higher risk of LRTIs than ones with CLP (IRR of CLP, 1.96 and IRR of CL, 2.32). To determine how cleft palate is linked to LRTIs, objective assessments of swallowing function and aspiration should be included in further studies.

This study has some limitations. First, the outcomes were reported by mothers. Although information on infections reported by their mothers has been used and validated, ${ }^{28,29}$ subjective reporting bias might have led to an overestimation of risk. Second, there was no information on the severity of orofacial clefts. For example, it is important to determine whether clefts involve the lip, alveolus (and primary palate), and/or secondary palate and whether the cleft phenotype is complete, incomplete, partial, unilateral, bilateral, or central. ${ }^{30}$ Further studies should include the information on the severity of orofacial clefts. Third, although this study included a history of surgery, the time relationship between outcome and surgery was unknown. Moreover, we could not collect details on surgery for cleft lip. As this study only included infants aged 1 year, they might not have been exposed to the risk of complications after primary cleft palate surgery. Indeed, 93.9\% of infants with CP did not undergo any surgery under general anesthesia. There is still a possibility that infants with CP may have an increased risk after 12 months of age. The current results might have underestimated the risk of LRTIs in infants with CP. Fourth, although the follow-up rate was relatively high, approximately $10 \%$ of the participants were lost to follow-up. Indeed, 25 infants with orofacial clefts were lost at 1 year of age. In addition, the previous JECS study indicated that worse infant health status was a risk factor for loss to follow-up during the first year postpartum. ${ }^{31}$ Hence, severe LRTI cases might have been lost to follow up in this study. Fifth, the sample size of infants with orofacial clefts was relatively small. In particular, the number of infants with CP was only 36 . Thus, the results of infants with $\mathrm{CP}$ might have been estimated with insufficient power. Finally, there was no information on preventive treatments for LRTIs. For example, a systematic review indicated the effects of palivizumab in significantly reducing the frequency of hospitalizations owing to respiratory syncytial virus infections. ${ }^{32}$ Further studies should also include information on preventive treatments, such as the use of palivizumab.

In conclusion, infants with CLP and CL were associated with an increased risk of LRTIs, but ones with $\mathrm{CP}$ were not. Further studies should, however, include objective assessments of swallowing function and aspiration accordingly.

\section{ACKNOWLEDGMENTS}

We would like to express our gratitude to all the participants of JECS and all staff members involved in data collection.

The Japan Environment and Children's Study Group: Members of the JECS Group as of 2020: Michihiro Kamijima (principal investigator, Nagoya City University, Nagoya, Japan), Shin Yamazaki (National Institute for Environmental Studies, Tsukuba, Japan), Yukihiro Ohya (National Center for Child Health and Development, Tokyo, Japan), Reiko Kishi (Hokkaido University, Sapporo, Japan), Nobuo Yaegashi (Tohoku University, Sendai, Japan), Koichi Hashimoto (Fukushima Medical University, Fukushima, Japan), Chisato Mori (Chiba University, Chiba, Japan), Shuichi Ito (Yokohama City University, Yokohama, Japan), Zentaro Yamagata (University of Yamanashi, Chuo, Japan), Hidekuni Inadera (University of Toyama, Toyama, Japan), Takeo Nakayama (Kyoto University, Kyoto, Japan), Hiroyasu Iso (Osaka University, Suita, Japan), Masayuki Shima (Hyogo College of Medicine, Nishinomiya, Japan), Youichi Kurozawa (Tottori University, Yonago, Japan), Narufumi Suganuma (Kochi University, Nankoku, Japan), Koichi Kusuhara (University of Occupational and Environmental Health, Kitakyushu, Japan), and Takahiko Katoh (Kumamoto University, Kumamoto, Japan).

Conflicts of interest: None declared.

Data availability: Data are unsuitable for public deposition due to ethical restrictions and legal framework of Japan. It is prohibited by the Act on the Protection of Personal Information (Act No. 57 of 30 May 2003, amendment on 9 September 2015) to publicly deposit the data containing personal information. Ethical Guidelines for Medical and Health Research Involving Human Subjects enforced by the Japan Ministry of Education, Culture, Sports, Science and Technology and the Ministry of Health, Labour and Welfare also restricts the open sharing of the epidemiologic data. All inquiries about access to data should be sent to: jecs-en@nies.go.jp. The person responsible for handling enquiries sent to this e-mail address is Dr Shoji F Nakayama, JECS Programme Office, National Institute for Environmental Studies. 
Funding: The Japan Environment and Children's Study was funded by the Ministry of the Environment, Japan. The findings and conclusions of this article are solely the responsibility of the authors and do not represent the official views of the aforementioned government.

\section{APPENDIX A. SUPPLEMENTARY DATA}

Supplementary data related to this article can be found at https:// doi.org/10.2188/jea.JE20200438.

\section{REFERENCES}

1. Mossey PA, Catilla EE, WHO Human Genetics Programme, WHO Meeting on International Collaborative Research on Craniofacial Anomalies (3rd: 2001: Bauru, Brazil). Global registry and database on craniofacial anomalies: report of a WHO Registry Meeting on Craniofacial Anomalies. Published online 2003. https://apps.who. int/iris/handle/10665/42840.

2. Natsume N, Kawai T, Kohama G, et al. Incidence of cleft lip or palate in 303738 Japanese babies born between 1994 and 1995. Br J Oral Maxillofac Surg. 2000;38(6):605-607.

3. Mossey PA, Little J, Munger RG, Dixon MJ, Shaw WC. Cleft lip and palate. Lancet. 2009;374(9703):1773-1785.

4. GBD 2016 Lower Respiratory Infections Collaborators. Estimates of the global, regional, and national morbidity, mortality, and aetiologies of lower respiratory infections in 195 countries, 1990 2016: a systematic analysis for the Global Burden of Disease Study 2016. Lancet Infect Dis. 2018;18(11):1191-1210.

5. Ministry of Health, Labour and Welfare. Patient Survey. Published 2017. Accessed March 26, 2020. https://www.mhlw.go.jp/toukei/ list/10-20.html.

6. Troeger CE, Khalil IA, Blacker BF, et al. Quantifying risks and interventions that have affected the burden of lower respiratory infections among children younger than 5 years: an analysis for the Global Burden of Disease Study 2017. Lancet Infect Dis. 2020; 20(1):60-79.

7. Amand C, Tong S, Kieffer A, Kyaw MH. Healthcare resource use and economic burden attributable to respiratory syncytial virus in the United States: a claims database analysis. BMC Health Serv Res. 2018;18(1):294.

8. Matsuo K, Palmer JB. Anatomy and physiology of feeding and swallowing: normal and abnormal. Phys Med Rehabil Clin N Am. 2008;19(4):691-707, vii.

9. Miller CK. Feeding issues and interventions in infants and children with clefts and craniofacial syndromes. Semin Speech Lang. 2011; 32(2):115-126.

10. Reid J, Kilpatrick N, Reilly S. A prospective, longitudinal study of feeding skills in a cohort of babies with cleft conditions. Cleft Palate Craniofac J. 2006;43(6):702-709.

11. Cushing AH, Samet JM, Lambert WE, et al. Breastfeeding reduces risk of respiratory illness in infants. Am J Epidemiol. 1998;147(9): 863-870.

12. Oddy WH, Sly PD, de Klerk NH, et al. Breast feeding and respiratory morbidity in infancy: a birth cohort study. Arch Dis Child. 2003;88(3):224-228.

13. Becker M, Svensson H, Källén B. Birth weight, body length, and cranial circumference in newborns with cleft lip or palate. Cleft Palate Craniofac J. 1998;35(3):255-261.

14. Wyszynski DF, Sarkozi A, Vargha P, Czeizel AE. Birth weight and gestational age of newborns with cleft lip with or without cleft palate and with isolated cleft palate. J Clin Pediatr Dent. 2003;27(2):185190.

15. Hviid A, Melbye M. The impact of birth weight on infectious disease hospitalization in childhood. Am J Epidemiol. 2007;165(7): 756-761.

16. Japanese Society of Oral and Maxillofacial Surgeons. Koshinretsu Kogairetsu Shinryo Guidelines. 2008. Accessed March 9, 2020. https://www.jsoms.or.jp/pdf/mg_cpf20080804.pdf.

17. Japan Society of Plastic and Reconstructive Surgery, Japan Society for Surgical Wound Care, Japan Society of Cranio-Maxillo-Facial Surgery. Keisei Geka Shinryo Guideline. Vol 6. KANEHARA; 2015.

18. Paine KM, Tahiri Y, Wes AM, et al. An Assessment of 30-Day Complications in Primary Cleft Lip Repair: A Review of the 2012 ACS NSQIP Pediatric. Cleft Palate Craniofac J. 2016;53(3):283289.

19. Takemura H, Yasumoto K, Toi T, Hosoyamada A. Correlation of cleft type with incidence of perioperative respiratory complications in infants with cleft lip and palate. Paediatr Anaesth. 2002;12(7): $585-588$.

20. Jama-Alol KA, Moore HC, Jacoby P, Bower C, Lehmann D. Morbidity due to acute lower respiratory infection in children with birth defects: a total population-based linked data study. $B M C$ Pediatr. 2014;14:80.

21. VanderWeele TJ author. Explanation in Causal Inference: Methods for Mediation and Interaction. Oxford University Press; 2015.

22. Kawamoto T, Nitta H, Murata K, et al; Working Group of the Epidemiological Research for Children's Environmental Health. Rationale and study design of the Japan environment and children's study (JECS). BMC Public Health. 2014;14:25.

23. Michikawa T, Nitta H, Nakayama SF, et al; Japan Environment and Children's Study Group. Baseline profile of participants in the Japan Environment and Children's Study (JECS). J Epidemiol. 2018;28(2): 99-104.

24. Mezawa H, Tomotaki A, Yamamoto-Hanada K, et al. Prevalence of congenital anomalies in the Japan Environment and Children's Study. J Epidemiol. 2019;29(7):247-256.

25. Zou G. A modified poisson regression approach to prospective studies with binary data. Am J Epidemiol. 2004;159(7):702-706.

26. Schempf AH, Kaufman JS. On the percent of excess risk explained. J Epidemiol Community Health. 2011;65(2):190; author reply 190.

27. Torgo L, Torgo ML. Package 'DMwR.' Comprehensive R Archive Network. Published online 2013.

28. Lain SJ, Roberts CL, Warning J, Vivian-Taylor J, Ford JB. A survey of acute self-reported infections in pregnancy. BMJ Open. 2011; 1(1):e000083.

29. Bánhidy F, Acs N, Puhó EH, Czeizel AE. Maternal acute respiratory infectious diseases during pregnancy and birth outcomes. Eur $J$ Epidemiol. 2008;23(1):29-35.

30. Allori AC, Mulliken JB, Meara JG, Shusterman S, Marcus JR. Classification of Cleft Lip/Palate: Then and Now. Cleft Palate Craniofac J. 2017;54(2):175-188.

31. Kigawa M, Tsuchida A, Matsumura $\mathrm{K}$, et al; Japan Environment and Children's Study (JECS) Group. Factors of non-responsive or lost-to-follow-up Japanese mothers during the first year post partum following the Japan Environment and Children's Study: a longitudinal cohort study. BMJ Open. 2019;9(11):e031222.

32. Andabaka T, Nickerson JW, Rojas-Reyes MX, Rueda JD, Bacic Vrca V, Barsic B. Monoclonal antibody for reducing the risk of respiratory syncytial virus infection in children. Cochrane Database Syst Rev. 2013;(4):CD006602. 